\title{
Mortalidade materna na Bahia no período de 2012 a 2016
}

\author{
Maternal mortality in Bahia in the period from 2012 to 2016 \\ Mortalidad materna en Bahía en el período de 2012 a 2016
}

Elenita Carmo Gois ${ }^{1}$, Carina Santos de Jesus ${ }^{1}$, Gleidiane Carmo da Conceição ${ }^{1}$, Josely Bruce dos Santos $^{2 *}$, Renato Barbosa Reis ${ }^{3}$.

\section{RESUMO}

Objetivo: Analisar as taxas de mortalidade materna no estado da Bahia no período de 2012 a 2016. Métodos: Estudo ecológico do tipo série temporal com utilização do Sistema de Informação Geográfica (SIG) para georreferenciamento dos óbitos maternos diretos e indiretos nas macrorregiões do estado da Bahia, a população do estudo foram os óbitos maternos registrados no estado da Bahia no período de 2012 a 2016 obtidos por meio do DATASUS, através do Sistema de Informação de Mortalidade e do Sistema de Informações de Nascidos Vivos. Resultados: Efetuou-se o cálculo das taxas de mortalidade materna geral para as macrorregiões do estado da Bahia e a média para o estado foi de 365,4/100.000N/V casos de óbitos, com ressalto para os anos de $2013779,4 / 100.000 \mathrm{~N} / \mathrm{V}$ e $2015656,9 / 100.000 \mathrm{~N} / \mathrm{V}$, a qual retrataram as maiores taxas no decorrer do período estudado. Conforme a distribuição espacial, os óbitos se concentraram nas regiões extremo sul, nordeste e norte. Conclusão: No decorrer dos anos as taxas de mortalidade materna com relação ao estado da Bahia mostraram-se em predisposição de declínio, mesmo diante das vagarosas medidas relacionadas às causas de morte, a literatura traz que, apesar de ter trilhado na direção para a diminuição da mortalidade materna, os princípios norteados para a duração da gestação são fatores importantes e ressalta um déficit na assistência de qualidade no período gestacional.

Descritores: Morte materna, Pré-natal, Mapeamento geográfico.

\section{SUMMARY}

Objective: To analyze the maternal mortality rates in the State of Bahia from 2012 to 2016. Methods: Ecological study of the temporal series type using the Geographic Information System (GIS) for georeferencing of direct and indirect maternal deaths in the macroregions of the state of Bahia. Bahia, the study population were maternal deaths recorded in the state of Bahia in the period from 2012 to 2016 obtained through DATASUS, through the Mortality Information System and the Information System for Live Births. Results: The general maternal mortality rates were calculated for the macroregions of the state of Bahia and the mean for the state was 365.4 / 100,000 N / V cases of deaths, with a rebound for the years of $2013779.4 / 100,000 \mathrm{~N} /$ $\mathrm{V}$ and $2015656.9 / 100,000 \mathrm{~N} / \mathrm{V}$, which showed the highest rates during the period studied. According to spatial distribution, deaths were concentrated in the extreme south, northeast and north regions. Conclusion: Over the years, maternal mortality rates in relation to the state of Bahia have shown a declining predisposition, even in the face of the slow measures related to the causes of death, the literature shows that, despite having walked in the direction of decline of maternal mortality, the guiding principles for the duration of gestation are important factors and highlights a deficit in quality care in the gestational period.

Descriptors: Maternal death, Prenatal care, Geographical mapping.

\footnotetext{
${ }^{1}$ Discentes do curso de graduação em enfermagem do Centro Universitário Jorge Amado, Salvador, Bahia.

'Enfermeira' Docente do curso em Enfermagem do Centro Universitário Jorge Amado, Salvador, Bahia.

*E-mail: joselybruce3@gmail.com

${ }^{3}$ Geógrafo, docente da Universidade Salvador, Salvador, Bahia.
} 


\section{RESUMEN}

Objetivo: Analizar las tasas de mortalidad materna en el estado de Bahía en el período de 2012 a 2016. Métodos: Estudio ecológico del tipo serie temporal con utilización del Sistema de Información Geográfica (SIG) para georreferenciación de los fallecimientos maternos directos e indirectos en las macrorregiones del estado de Bahía La población del estudio fue las muertes maternas registradas en el estado de Bahía en el período de 2012 a 2016 obtenidos por medio de DATASUS, a través del Sistema de Información de Mortalidad y del Sistema de Informaciones de Nacidos Vivos. Resultados: Se realizó el cálculo de las tasas de mortalidad materna general para las macrorregiones del estado de Bahía y la media para el estado fue de 365,4 / $100.000 \mathrm{~N} / \mathrm{V}$ casos de óbitos, con rebaja para los años de 2013 779,4 / 100.000N/V y 2015656,9 / 100.000N / V, la cual retrataron las mayores tasas durante el período estudiado. Conforme a la distribución espacial, las muertes se concentraron en las regiones extremo sur, nordeste, norte. Conclusión: En el transcurso de los años las tasas de mortalidad materna con relación al estado de Bahía se mostraron en predisposición de declinación, incluso ante las vagarosas medidas relacionadas con las causas de muerte, la literatura trae que, a pesar de haber trillado en la dirección hacia la disminución de la mortalidad materna, los principios orientados a la duración de la gestación son factores importantes y resalta un déficit en la asistencia de calidad en el período gestacional.

Palavras-clave: Muerte Materna, Atención Prenatal, Mapeo Geográfico.

\section{INTRODUÇÃO}

A mortalidade materna, é caracterizada como a morte durante a gravidez ou no prazo de 42 dias após a gestação, engloba a saúde pública de forma geral. É um potente indicador em países em desenvolvimento por representar uma grande parcela dos óbitos registrados (DIAS et al., 2015).

Os óbitos maternais encontram-se associadas a razões obstétricas diretas e indiretas. Tais problemas tendem a manifestar-se no decorrer da fase gestacional ou perante o puerpério. Podendo apresentar se tal como doença hipertensiva específica da gravidez (DHEG), hemorragias, aborto, infecções entre outras. . (BRASIL, 2012a).

Entre 1990 e 2015 a redução na razão de mortalidade materna no Brasil foi de 143 para 62 óbitos maternos por 100 mil nascidos vivos, o que representou uma diminuição de $56 \%$. Esta redução tem sido reconhecida, ao destacar que houve avanços significativos desde a década de 90 nas políticas públicas de saúde para as mulheres (OMS, 2015).

De acordo com dados do Sistema de Informação sobre Mortalidade (SIM), em 2015, o Brasil registrou 1.738 casos de morte materna, que engloba óbitos causados por problemas relacionados à gravidez ou ao parto ou ocorridos até 42 dias depois. Em 2016, foram registrados 1.463 casos, uma queda de 16\% em relação ao ano anterior.

As estratégias de redução das taxas de mortalidade materna no país, foi instituído em 1983, o Programa de Assistência Integral à Saúde da Mulher (PAISM), expandindo as ações de saúde prestadas à população feminina. E no ano 2000, o Ministério da Saúde (MS) lançou o Programa Nacional de Humanização ao Prénatal e Nascimento (PHPN), que apontou critérios para uma assistência de qualidade destinada às gestantes. Já em 2011, constrói-se a Rede Cegonha como nova proposta de organização e planejamento da rede de atenção ao parto e nascimento no Brasil (BRASIL, 2011).

Como $92 \%$ das causas de óbito materno estão relacionadas às causas evitáveis principalmente, por hipertensão, hemorragia ou infecções, surge a relevância da assistência pré-natal iniciar precocemente e de maneira periódica de modo a promover a saúde e evitar precocemente complicações que possam levar a mortalidade materna (NOGUEIRA e OLIVEIRA, 2017). Dados revelam que a região Nordeste ocupa a segunda posição no ranking de baixa cobertura da assistência pré-natal no Brasil com $34,5 \%$ atrás da região Norte com $26,5 \%$ (SHIMIZU e LIMA, 2009). 
No Brasil, o pré-natal é considerado como de baixa eficácia, e a atenção ao parto e ao puerpério são consideradas etapas esquecidas, algumas gestantes devido a inúmeras questões de ordem social, não realizam o pré-natal o que contribui para o aumento da taxa de mortalidade materna (NOGUEIRA e OLIVEIRA, 2017). Mediante o exposto, o objetivo deste estudo é avaliar as taxas de mortalidade materna na Bahia no período de 2012 a 2016.

\section{METODOLOGIA}

Trata-se de um estudo ecológico do tipo série temporal com utilização do Sistema de Informação Geográfica (SIG) para georreferenciamento dos óbitos maternos diretos e indiretos nas macrorregiões do estado da Bahia. A população do estudo foi da macrorregião do extremo sul, residentes no estado da Bahia no período de 2012 a 2016.

Os dados foram coletados por meio da base de dados do Sistema Único de Saúde, o DATASUS, através do Sistema de Informação de Mortalidade (SIM) para o período de estudo citado. Os óbitos foram coletados por ano, ocorrência, pesquisados com base no Capítulo XVI da lista de Classificação Internacional de Doenças (CID-10) que corresponde à algumas aos óbitos maternos por causas diretas e indiretas a partir da faixa etária 10 a 59 anos. Foram coletados também os dados referentes aos nascidos vivos, registrados no Sistema de Informações de Nascidos Vivos (SINASC), por meio da ferramenta para tabulação de dados (TABNET) na opção Estatísticas vitais do DATASUS, através do grupo nascidos vivos, por local de residência, macrorregião e ano, para o cálculo da taxa de mortalidade no período compreendido entre 2012 e 2016 .

As variáveis disponíveis no DATASUS utilizadas neste estudo foram: macrorregião de saúde de ocorrência, ano, faixa etária e o capítulo XV, da Classificação Internacional de Doenças (CID 10). Para estimar a taxa de mortalidade materna nas macrorregiões de saúde da Bahia foi utilizada a seguinte fórmula: calculouse o número de óbitos maternos por causas diretas e indiretas, dividiu-se pelo número de nascidos vivos para cada ano e multiplicou-se por cem mil habitantes.

As taxas de mortalidade foram calculadas para cada ano no período citado e compiladas em tabelas e gráficos para análise dos dados e discussão dos mesmos. Os dados foram apresentados sob a forma de taxas de mortalidade, estes foram processados e as tabelas geradas com o auxílio do Microsoft Excel 2013 possibilitando sua interpretação para o período de estudo.

Utilizou-se o Sistema de Informação Geográfica (SIG), para georrefenciamento das taxas de mortalidade materna por macrorregião de saúde na elaboração dos mapas dos municípios, por ano, do estado da Bahia, entre os anos de 2012 e 2016. O georreferenciamento ou geoprocessamento dos dados de mortalidade foi realizado tendo como unidades espaciais os municípios e em seguida as macrorregiões de saúde. Realizouse a geolocalização do quantitativo de óbitos maternos para o grupo das causas através do Software ArcGIS 10.1 utilizando o código do município como elo para vinculação entre a tabela construída no Microsoft Excel 2013 e a malha digital (mapa) do estado da Bahia gerado pelo Software supracitado para a análise da distribuição das taxas de mortalidade nas macrorregiões de saúde do estado da Bahia. Os resultados foram apresentados através de mapas, estes com intervalos de classe contendo as taxas de mortalidade calculadas por ano e a taxa cumulativa para o período em análise.

\section{RESULTADOS}

A Figura 1 traz a distribuição espacial da taxa de mortalidade materna no estado da Bahia no período de 2012 a 2016, nota-se que o extremo sul ao longo dos cincos anos tem as maiores taxas de mortalidade diminuindo apenas em 2014 e 2016 comparados a outras macrorregiões, representadas por tons amarelo e vermelho no mapa, houve uma redução significativa na taxa de mortalidade anual, sem contar os casos subnotificados que não chegam a ser contabilizados. Em 2012 houve uma redução na quantidade de municípios do extremo sul, e com isso uma diminuição no número de mortes maternas. Em 2013 o destaque se deu pelo aumento significativo nas taxas de mortalidade do centro-norte e o Sudoeste as taxas identificadas. 
Figura 1. Distribuição espacial das taxas de mortalidade materna segundo as macrorregiões de saúde na Bahia entre 2012 a 2016. Salvador, Bahia, Brasil.

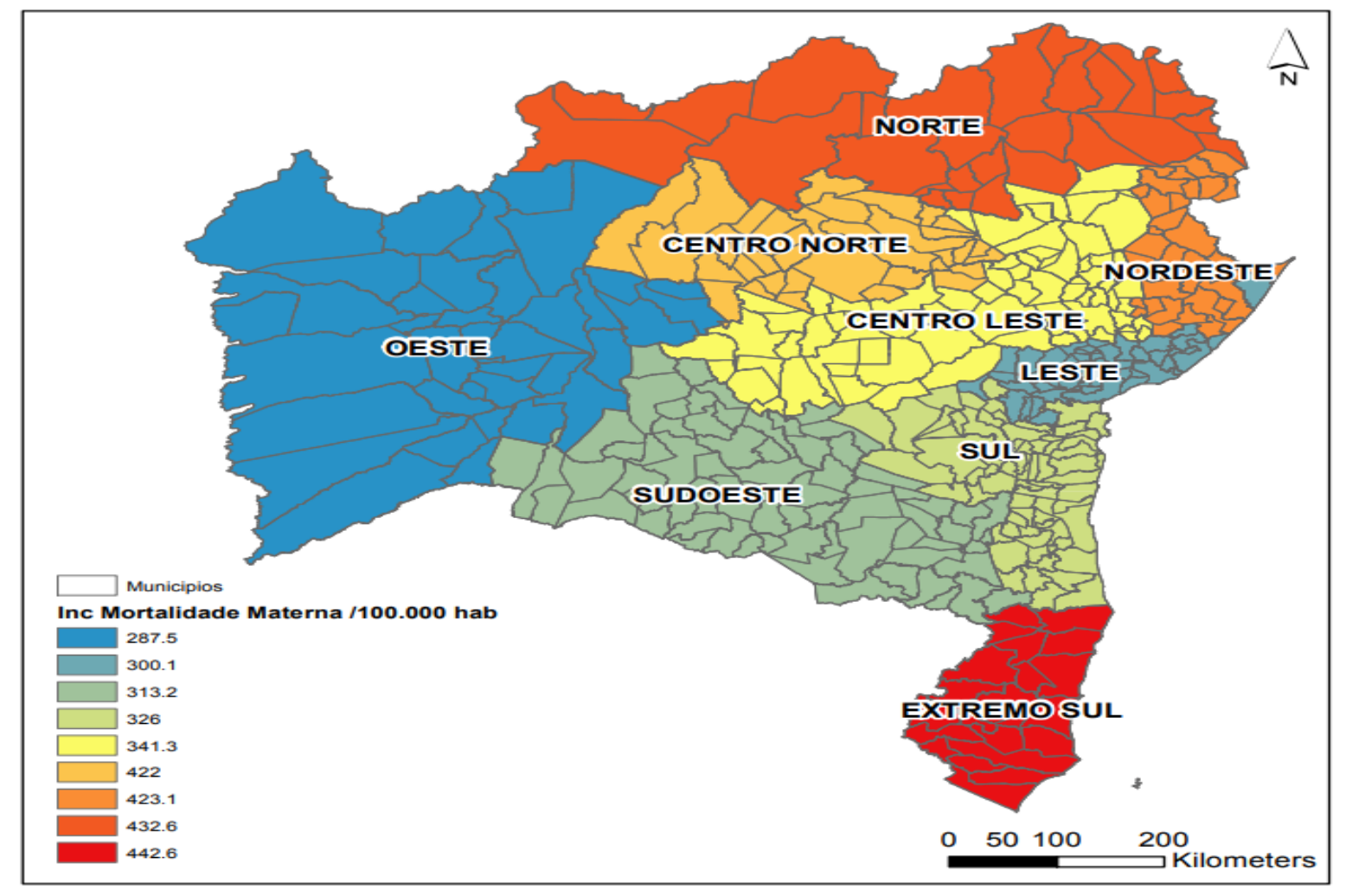

Fonte: Sistema de Informação Geográfica (SIG).

Tabela 1. Taxa de mortalidade por ano segundo as macrorregiões de saúde na Bahia no período de 2012 a 2016. Salvador, Bahia, Brasil.

\begin{tabular}{|c|c|c|c|c|c|}
\hline \multicolumn{6}{|c|}{ Taxa de mortalidade ${ }^{*}$} \\
\hline & 2012 & 2013 & 2014 & 2015 & 2016 \\
\hline \multicolumn{6}{|l|}{ REGIÃO } \\
\hline $\begin{array}{l}\text { Centro- } \\
\text { leste }\end{array}$ & 41,9 & 71,5 & 74,4 & 80,7 & 72,8 \\
\hline $\begin{array}{l}\text { Centro- } \\
\text { norte }\end{array}$ & 53 & 161 & 55,1 & 79,4 & 73,5 \\
\hline Extremo sul & 105,1 & 135,8 & 54,3 & 91,7 & 55,7 \\
\hline Leste & 61,8 & 64,7 & 67,6 & 56 & 50 \\
\hline Nordeste & 74,3 & 86,5 & 89,4 & 78,6 & 94,3 \\
\hline Norte & 118,9 & 74,6 & 85,8 & 88,2 & 65,1 \\
\hline Oeste & 49,3 & 28,7 & 82,4 & 62,7 & 64,4 \\
\hline Sudoeste & 68,1 & 114,1 & 13 & 55,3 & 62,7 \\
\hline Sul & 81,3 & 42,5 & 87,6 & 64,3 & 40,3 \\
\hline
\end{tabular}

Fonte: Sistema de informação de mortalidade. 
No que se refere a distribuição espacial dos óbitos maternos por macrorregião no estado da Bahia no período de 2014 nota-se que o gráfico 1 destaca que a região Nordeste e região Sul apresentaram a maior taxa, houve um aumento significativo na região Oeste e a menor taxa na região Sudoeste, houve uma redução nas taxas identificadas para cada $100.000 \mathrm{NV}$ notou-se também que algumas regiões tiveram registro maior a /100.000NV.

Gráfico 1. Tendência de variação da mortalidade materna nas macrorregiões de saúde na Bahia no período entre 2012 a 2016. Salvador, Bahia, Brasil.

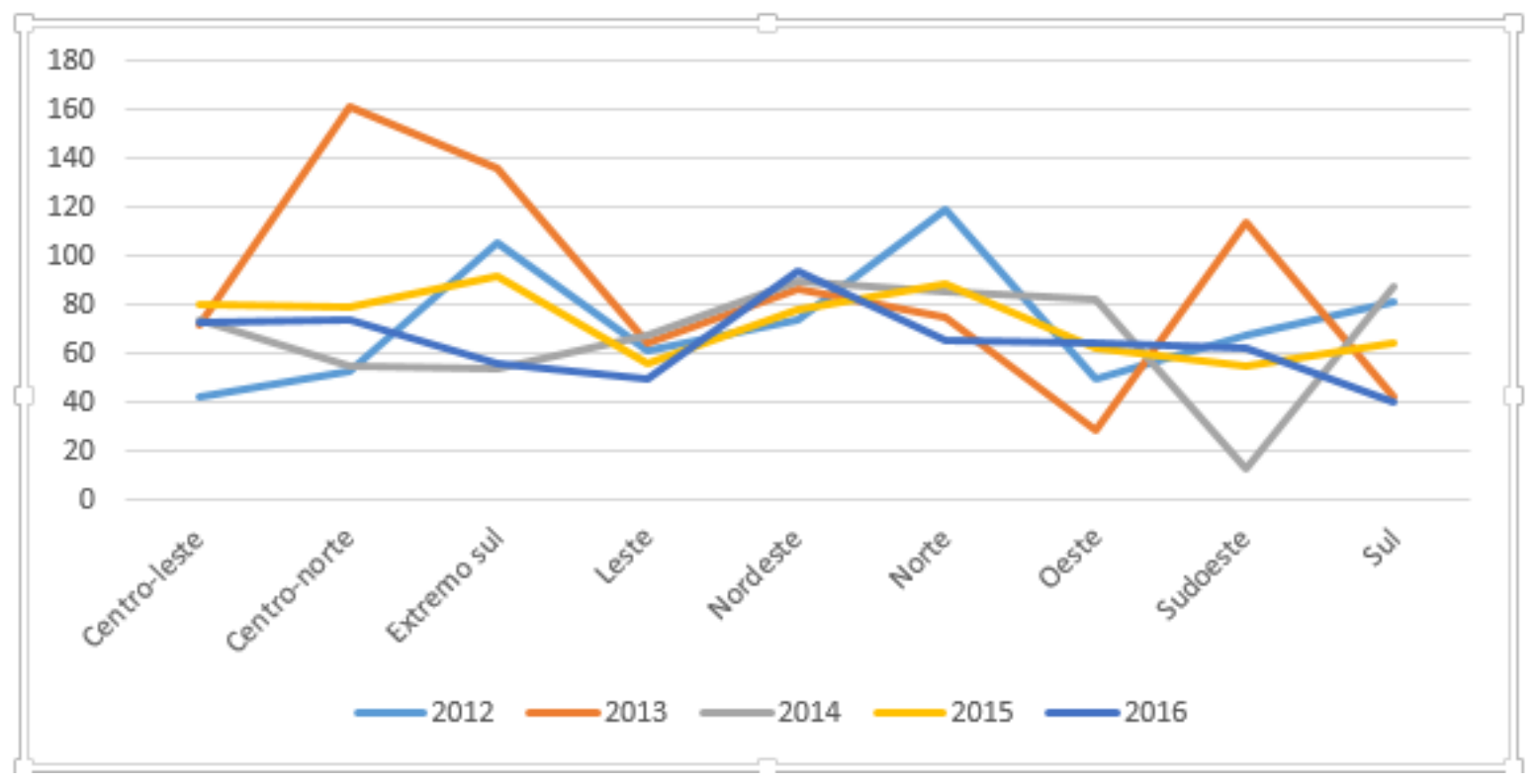

Fonte: Dados da pesquisa, 2018.

O Gráfico 1 também apresenta os óbitos maternos no ano de 2015 por macrorregião no estado da Bahia, o destaque desse período identificou que no Extremo Sul voltou a ter a maior taxa representada pela cor vermelha na Figura 1, isso corresponde a baixa cobertura na assistência à saúde, obteve aumento da taxa de mortalidade identificada para 91,7/100.000NV.

No ano de 2014 o gráfico expõe os óbitos maternos por macrorregião do estado da Bahia, no ano de 2016 representado por um aumento de taxa na região Nordeste e a menor taxa identificada na região Sul, portanto pode-se observar que se manteve a distribuição por todas as regiões, contudo esse foi o ano generalizado que houve a menor taxa referente a todas as regiões no período de 2012 a 2016.

\section{DISCUSSÃO}

Ao longo dos cinco anos no extremo sul, notou-se que as taxas de mortalidade materna apresentaram tendência de variação. Com maiores taxas de óbitos maternos na faixa etária entre 20 a 29 anos, mulheres negras, com escolaridade ignorada e estado civil, solteira. É importante salientar que a maioria das causas diretas poderia ser evitável. Já as indiretas, precisam ser avaliadas com maior importância, pois essas mulheres já portadoras de doenças devem enquanto gestantes de risco, serem encaminhadas para atendimento específico (DIAS et al., 2015)

As mulheres negras e solteiras encontram-se em situação de vulnerabilidade, são as que apresentam níveis de desnutrição mais altos, escolaridade e baixa renda, estão concentradas em locais de difícil acesso aos serviços de saúde, o que aumenta o risco para a mortalidade materna tanto por causas diretas quanto indiretas (RIBEIRO et al., 2010). 
De acordo com Cunha et al. (2013), o Brasil possui a segunda maior população negra do mundo, com quase $50 \%$ de seu povo auto declarado como afrodescendente (pardos ou pretos) como resultado do processo histórico da escravatura iniciado a partir do século XVI. A construção social das desigualdades entre brancos e negros interfere na forma de viver destas pessoas e faz-se evidente nas disparidades das condições de existência entre um grupo e outro. Para Bastos (2000) as condições socioeconômicas existentes no Brasil são desfavoráveis à população negra. Lopes (2004) revela que os negros, além de ocuparem lugares desiguais na sociedade, também possuem experiências desiguais ao nascer, viver, adoecer e morrer.

Na macrorregião do extremo sul as causas obstétricas diretas são as que têm maior influência nas mortes maternas, porém as causas indiretas, que são provenientes de doenças graves que se associam à gestação, influenciam de forma importante na taxa de mortalidade. Ainda de acordo com Dias et al. (2015), no Brasil, as causas obstétricas diretas correspondem por $66,7 \%$ das mortes maternas e suas principais causas são: as doenças hipertensivas, hemorragias, as complicações do aborto e as infecções puerperais, com relação direta a fatores socioeconômicos.

De acordo com Cunha et al. (2012), por ano quase 600 mil mulheres em todo o mundo vão a óbito, vítimas de complicações na gravidez. Dessas 95\% aconteceram em países em desenvolvimento e subdesenvolvidos e as outros $5 \%$ em países desenvolvidos.

O Ministério da Saúde (2012b) divulgou dados sobre a mortalidade materna por tipo de causa obstétrica direta como: Aborto, Hemorragia, Hipertensão, Infecção puerperal e outras causas obstétricas diretas, considerando as desigualdades relativas à mortalidade materna, as Regiões Norte, Nordeste e Centro-Oeste apresentavam percentuais por causas diretas acima da média nacional, 72,1\%, 70,3\% e 67,2\%, respectivamente.

No Brasil óbitos maternos são causados em dois terços por causa obstétrica direta caracterizado pela baixa qualidade da assistência e de planejamento familiar oferecido a população feminina brasileira. Segundo o Ministério da saúde (MS) acredita-se que aproximadamente 95\% dessas mortes seriam impedidas por um atendimento eficaz e qualidade assistencial para as gestantes durante o ciclo gravídico, parto e pós-parto (BOTHELHO et al., 2015).

De acordo Guimarães et al. (2017), em países das Américas, a diferença entre países crescidos e em crescimento torna-se óbvio. Estados Unidos e Canadá apontam padrões menores a 11 óbitos maternos para 100.000 nascidos vivos, ao mesmo tempo em que países como Bolívia e Peru atingem mais de 200 óbitos. Aproximadamente 15.000 mulheres na América Latina, falecem por ano devido a problemas na gestação, parto ou puerpério. Os indícios precisos acerca da mortalidade materna apontam que as soluções obtidas pela maior parte dos países não serão eficazes para a execução da Meta de Desenvolvimento do Milênio (FERNANDES et al., 2015).

Outro dado relevante, é que no estudo de Menezes et al. (2015) revela índices elevados de mortalidade materna em partos cesarianos no Brasil o que permite a redução de óbitos materno no país resultando em altas taxas de crescimento em todas as regiões. De acordo com MS esse percentual não deve ser maior que $15 \%$ do total de partos, pois poderá acarretar para a mãe um risco de 3,5 vezes na probabilidade de óbito e cinco vezes mais de contrair uma infecção hospitalar.

Diante de taxas elevadas de mortalidade materna, o Ministério da saúde implantou por meio da portaria 653, que caracterizou morte materna como uma ação compulsória, direcionando que esse evento seja obrigatório a notificação dos casos. No Brasil foi lançado o projeto como comitê de mortalidades e vigilância epidemiológica para descobrir óbitos materno em mulheres reprodutivas e diminuir os graves problemas de sub-registro (ÁFIO et al., 2014).

Ainda que se tenha percebido uma redução das taxas de mortalidade materna durante os anos 2009 e 2012 neste estudo, para Lopes et al. (2016), ocorreu um crescimento na soma de mortes em 2013, o que arriscasse ter acontecido mediante uma melhor apuração de óbitos materno nesse ano. Lopes et al. (2016), ressalta causas que podem motivar o fato das subnotificações e sub-registro, tal como dados ilusórios nos 
registros e a insciência e incapacidade dos revisores. A correta complementação das informações de óbitos seria um coeficiente fundamental para evitar essa falha.

Deste modo, todos os acontecimentos durante a gravidez devem ser registrados para uma avaliação de qualidade durante pré-natal. A qualidade da assistência do pré-natal vai refletir de forma positiva na redução de taxa de mortalidade materna, diversos autores relatam em estudos recentes a assiduidade na vigilância em mulheres no terceiro trimestre de gestação, o intervalo reduzido de uma consulta para outra, por ser o de maior risco para as complicações gestacionais (CARVALHO et al., 2015).

De acordo com pesquisa realizada no Brasil é relativo com o aumento de valores da cobertura assistência de pré-natal comparando com resultado de todas as regiões do país da população feminina em fase reprodutiva e social. Diante do contexto a adaptação da assistência ainda é baixa apenas $75,8 \%$ das gestantes começaram o acompanhamento pré-natal com 37 semanas de gestação enquanto $73,1 \%$ alcançaram a quantidade de consulta adequada para a idade gestacional (VIELLAS et al., 2014).

Isto demostra a fragilidade da assistência, na identificação e ações prévias na assistência inicial. Dessa forma, possibilitar a estas usuárias, no momento em que for preciso, o ingresso à demais áreas de atenção, através de linhas de referência e contra referência, mediante forma de atividades elaboradas através dos municípios e estado, a fim de um apoio integro ao bem-estar da mulher no período gestacional puerperal (BRASIL, 2012c).

Independentemente da diminuição nas taxas da mortalidade materna já obtidas pelo Brasil, o país não conseguiu atingir $5^{\circ}$ meta do milênio, a restrição em $3 / 4$ das taxas entre 1990 e 2015 . Conforme estimação da secretaria de Vigilância em Saúde do Ministério da Saúde, a razão de mortalidade materna era de 141 por 100.000 nascidos vivos em 1990 e decaiu para 68 por 100.000 nascidos vivos em 2010. Por volta de janeiro e setembro de 2011 , a mortalidade materna reduziu $21 \%$. Houve 1.038 mortes por intermédio de problemas na gestação e no parto, versus 1,317 no mesmo intervalo de 2010. A meta era alcançar 35 óbitos por 100.000 nascidos vivos em 2015 (BRASIL, 2013).

Ainda de acordo Guimarães et al. (2017), independente da diminuição da mortalidade materna, esta pesquisa comprova as diferenças entre os territórios e confirma, através de fatores apontados, a fragilidade de determinados grupos de habitantes. Perante isso, percebe-se que, apesar de ter trilhado na direção para a diminuição da mortalidade materna, os princípios norteados para esse âmbito até então dispõem de vastos problemas a serem atingidos.

\section{CONCLUSÃO}

O presente estudo aponta uma análise das taxas de mortalidade materna por nascidos vivos, a começar da qualidade da assistência pré-natal prestada no estado da Bahia ao longo de 5 anos, que além dos empenhos usados, as taxas sustentaram -se altas. A assistência pré-natal, como aponta a literatura pode não impedir os principais problemas do parto e puerpério, razões primordiais de mortalidade materna, porém a atenção qualificada consegue mudar e ajudar no prognostico materno antecipado. Este estudo também apresentou as causas pertinentes ao óbito, considerando fatores intersetoriais e de assistência médica realizada às grávidas e/ou as puérperas. Apurou-se que, em praticamente seu total, as situações das concepções e óbitos aconteceram em meio hospitalar. Entende-se que as razões de mortalidade materna no Brasil apontam para diferenças regionais, expondo uma realidade que carece de ações efetivas no âmbito da saúde, com o objetivo que se disponha de padrões satisfatórios no âmbito materno-infantil.

\section{REFERÊNCIAS}

1. AFIO ACE, ARAÚJO MAL, ROCHA AFB et al. Óbitos materno: necessidades de repensar estratégias de enfrentamento. Rev Rene, 2014; 15(4):631-8.

2. BASTOS FI. A Feminização da epidemia da Aids no Brasil: determinantes estruturais e alternativas de enfrentamento. Saúde Sexual e Reprodutiva, 200; 3:1-27. 
3. BOTHELHO NM, SILVA IFMM, TAVARES JR et al. Causas de morte materna no Estado do Pará, Brasil. Revista Brasileira Ginecológica Obstétrica, 2014; 36(7): 290-295.

4. BRASIL. Ministério da Saúde Secretaria de Vigilância em Saúde Departamento de Análise de Situação em Saúde. Brasília: Saúde Brasil, 2012c.

5. BRASIL. Ministério da saúde. Guia de Apoio para o Alcance das Metas, agenda de Compromissos dos Objetivos de Desenvolvimento do Milênio. Brasília, 2013. 56-60p

6. BRASIL. Ministério da saúde. Assistência integral à Saúde da mulher: bases de ação programática. Brasília: Ministério da Saúde, 1984.26p.

7. BRASIL. Ministério da Saúde. Boletim Epidemiológico. Brasília: Secretaria de Vigilância em Saúde, 2012a.

8. BRASIL. Ministério da Saúde. Secretaria de Atenção à Saúde. Programas de atenção à saúde da mulher Brasília: Ministério da saúde, 2012b.

9. CARVALHO ML, ALMEIDA CAL, MARQUES AKL et al, Prevenção da mortalidade materna no pré-natal: uma revisão integrativa. Revista Interd., 2015; 8(2): 178-184.

10. CUNHA GM, MORAES LRS, LIMA AGD, MATTOS PSMS, FREDIANI D. A Prevalência da infecção por enteroparasitas e sua relação com as condições socioeconômicas e ambientais em comunidades extrativistas do município de Cairu - Bahia. Revista Eletrônica de Engenharia Civil, 2013; 7(2): 27-36.

11. CUNHA MA, MAMEDE MV, DOTTO LMG et al. Assistência Pré-Natal Por Profissionais De Enfermagem No Município De Rio Branco, Acre, Amazônia. Revista Baiana de Saúde Pública, 2012; 36(1):174-190.

12. DIAS JMG, Oliveira APS, CIPOLOTTIR et al. Mortalidade materna. Revista Med Minas Gerais, 2015; 25(2): 173179.

13. FERNANDES B, PRUDÊNCIO PS, NUNES FBBF et al. Pesquisa epidemiológica dos óbitos maternos e o cumprimento do quinto objetivo de desenvolvimento do milênio. Rev Gaúcha Enfermagem, 2015; 36(esp): $192-$ 9.

14. GUIMARÃES TA, ROCHA AJSC, RODRIGUES WB et al. Mortalidade Materna no Brasil entre 2009 e 2013. Rev Pesquisa Saúde, 2017; 18(2): 81-85.

15. LOPES F. Experiências desiguais ao nascer, viver, adoecer e morrer: tópicos em saúde da população negra no Brasil. Brasília: Ministério da Saúde, 2004.

16. LOPES FBT, MORAES MS, RODRIGUES APRA et al. Mortes Maternas por Causas Sensíveis a Atenção Primária. Ciências Biológicas e da Saúde, 2016; 3(3): 201-214.

17. MENEZES MLN, BEZERRA JFO, BEZERRA JFO. Perfil epidemiológico dos óbitos maternos em hospital de referência para gestação de alto risco. Rev Rene, 2015; 16(5);714-21.

18. NOGUEIRA LDP, OLIVEIRA GS. Assistência Pré-Natal Qualificada: As Atribuições Do Enfermeiro - Um Levantamento Bibliográfico. Rev Enferm Atenção Saúde, 2017; 6(1):107-119.

19. RIBEIRO PM, SIMÃO AB, CAETANO AC et al. Perfis de Vulnerabilidade Feminina ao HIV/aids em Belo Horizonte e Recife: comparando brancas e negras. Saúde Soc. São Paulo, 2010; 19(2): 21-35.

20. SHIMIZU HE, LIMA MG. As Dimensões Do Cuidado Pré-Natal Na Consulta De Enfermagem. Rev Bras. Enferm, 2009; 62(3): 387-92.

21. VIELLAS EL, DOMINGUES RMSM, DIAS MAB et al. Assistência do pré-natal do Brasil. Caderno de Saúde Pública, 2014; 30(Suppl 1): S85-S100. 